\title{
Search on Seeds for Drug Discovery from Medicinal Plants
}

\author{
Hiroshi Morita*
}

Faculty of Pharmaceutical Sciences, Hoshi University, Ebara 2-4-41 Shinagawa-ku, Tokyo 142-8501, Japan; E-mail:moritah@hoshi.ac.jp

Plants and Plants derived preparations have been employed in the treatment of numerous and diverse medical conditions. Plants remain to this day one of the most important sources of biological active compounds and continue to provide drug discovery seeds. During our search for active ingredients from medicinal plants through collaboration works in Malaysia and Indonesia, we have isolated various kinds of alkaloids and terpenoids with antitumor, vasorelaxant, and antiplasmodial activities, and inhibiting activity of lipid droplets accumulation as new drug discovery seeds.

In the field of drug and drug development, chirality is very important since the two enantiomers of a chiral drug generally possess different pharmaceutical activities. There are many methods of determining the absolute configuration (AC) of a chiral molecule, both directly or using chiral auxiliaries, and by empirical or non-empirical methods. The AC of some natural products in this work was assigned by circular dichroism (CD) exciton chirality, X-ray crystallography method, or by comparing the Time Dependent Density Functional Theory (TDDFT) calculated CD spectra with the experimental one. In this paper we describe the isolation, structure elucidation, and biological activity of some natural products from tropical medicinal plants as new drug discovery seeds. And we also mention the method for assignment of AC using calculated CD spectra.

Keywords: Alkaloids, Terpenoids, Biological activity, absolute configuration, circular dichroism (CD) spectrum. 